\title{
Correction
}

Concerning the paper:

\section{Characterization of polyethylene networks based on the jointed description of melting, swelling and deformation}

H.-G. Kilian, K. Unseld, E. Jaeger, J. Müller, and B. Jungnickel

Abteilung Experimentelle Physik, Universität Ulm, Ulm, F.R.G., and

Deustsches Kunststoff-Institut, Darmstadt, F.R.G.

Coll \& Polym Sci 263:607 (1985)

We give the correct notation of the Equations (28), (30), (33), (34), (35), (36), and (41).

$$
\begin{aligned}
-\frac{\Delta S_{\mathrm{mix}}}{k_{B}}= & -\frac{\Delta S_{\mathrm{mix}}^{i}}{k_{B}}+n_{p}(K-1) \ln \left(\varphi_{p}\right) \\
& +n_{p} K \ln (Z K)+n_{L} \ln Z \\
\frac{\Delta \mu_{\mathrm{mix}}^{L}}{k_{B} T}= & -\frac{1}{k_{B}}\left(\frac{\partial \Delta S_{\mathrm{mix}}}{\partial n_{L}}\right)_{T, P} \\
= & \ln \left(1-\varphi_{p}\right)+\left(1-\frac{y_{L}}{y_{p}}\right) \varphi_{p} \\
& +n_{p}\left(\frac{\partial K}{\partial n_{L}}\right)_{T, P}\left\{\ln \left(\varphi_{p}\right)+\ln (Z K)\right\} \\
& +(K-1) n_{p}\left(\frac{\partial \varphi_{p}}{\partial n_{L}}\right)_{T, P} \varphi_{p}^{-1} \\
& +\frac{1}{Z}\left\{n_{p}\left(\frac{\partial K}{\partial n_{L}}\right)_{T, P} Z+K n_{p}\left(\frac{\partial Z}{\partial n_{L}}\right)_{T, P}\right\} \\
& +\ln (Z)+n_{L}\left(\frac{\partial Z}{\partial n_{L}}\right)_{T, P}
\end{aligned}
$$

$$
\begin{aligned}
n_{p}\left(\frac{\partial K}{\partial n_{L}}\right)_{T, P}= & -\frac{y_{L} \varphi_{p}^{2 / 3}}{3 y_{p}}\left(\varphi_{p}^{-1 / 3}\right. \\
& \left.-\varphi_{p}^{2 / 3}\right)\left(I_{1} I_{m 1}\right)^{-1 / 2}
\end{aligned}
$$

$$
\begin{aligned}
n_{p}\left(\frac{\partial Z}{\partial n_{L}}\right)_{T, P}= & -Z^{2}\left\{\varphi_{p} n_{p}\left(\frac{\partial K}{\partial n_{L}}\right)_{T, P}\right. \\
& \left.+(K-1) n_{p}\left(\frac{\partial \varphi_{p}}{\partial n_{L}}\right)_{T, P}\right\}
\end{aligned}
$$

$$
\begin{aligned}
n_{L}\left(\frac{\partial Z}{\partial n_{L}}\right)_{T, P}= & -Z^{2}\left\{\varphi_{p} n_{L}\left(\frac{\partial K}{\partial n_{L}}\right)_{T, P}\right. \\
& \left.+(K-1) n_{L}\left(\frac{\partial \varphi_{p}}{\partial n_{L}}\right)_{T, P}\right\}
\end{aligned}
$$

$$
\begin{aligned}
n_{L}\left(\frac{\partial K}{\partial n_{L}}\right)_{T, P}= & -\frac{\varphi_{p}^{1 / 3}}{3}\left(1-\varphi_{p}\right)\left(\varphi_{p}^{-1 / 3}\right. \\
& \left.-\varphi_{p}^{2 / 3}\right)\left(I_{1} I_{m 1}\right)^{-1 / 2}
\end{aligned}
$$

$$
\begin{aligned}
\frac{\Delta \mu_{\lambda}^{L}}{k_{B} T} & =\frac{1}{k_{B} T}\left(\frac{\partial W}{\partial n_{L}}\right)_{T, P} \\
& =\frac{y_{L}}{y_{p}}\left(\frac{\lambda_{m o}}{\lambda_{m 1}}\right)^{2}\left(\frac{1}{1-\eta_{3}^{1 / 2}}-a \phi_{3}^{1 / 2}\right) \varphi_{p}^{1 / 3}
\end{aligned}
$$

